\title{
Pancreatic outflow obstruction as the critical event for human gall stone induced pancreatitis
}

\author{
M M Lerch, H Weidenbach, CA Hernandez, G Preclik, G Adler
}

\begin{abstract}
Opie suggested in 1901 that a common channel between the pancreatic duct and the common bile duct is created when a gall stone becomes impacted at the duodenal papilla. He proposed that bile would regurgitate into the pancreas and trigger pancreatitis. The case is reported of a 22 year old woman with an impacted stone at the duodenal papilla creating a common channel. The patient suffered from acute pancreatitis. Three days before the onset of pancreatitis, however, a T drain had been inserted into the common bile duct from which bile had been flowing freely and continuously. Moreover, amylase activity in fluid from the $T$ drain was $49000 \mathrm{U} / \mathrm{l}$ at the onset of pancreatitis pointing to reflux of pancreatic juice into the biliary tract. The amylase activity in bile decreased rapidly after endoscopic papillotomy and retrieval of the stone. The events participating in the development of acute gall stone induced pancreatitis in this patient with a common channel situation permitted reflux of pancreatic juice into the biliary tract rather than bile into the pancreas. Impairment of pancreatic outflow by a gall stone was probably the primary triggering event, rather than the regurgitation of bile into the pancreas. Preventive or therapeutic treatment in gall stone pancreatitis should be aimed at the urgent restoration of pancreatic flow rather than at the prevention of a hypothetical bile reflux.
\end{abstract}

(Gut 1994; 35: 1501-1503)

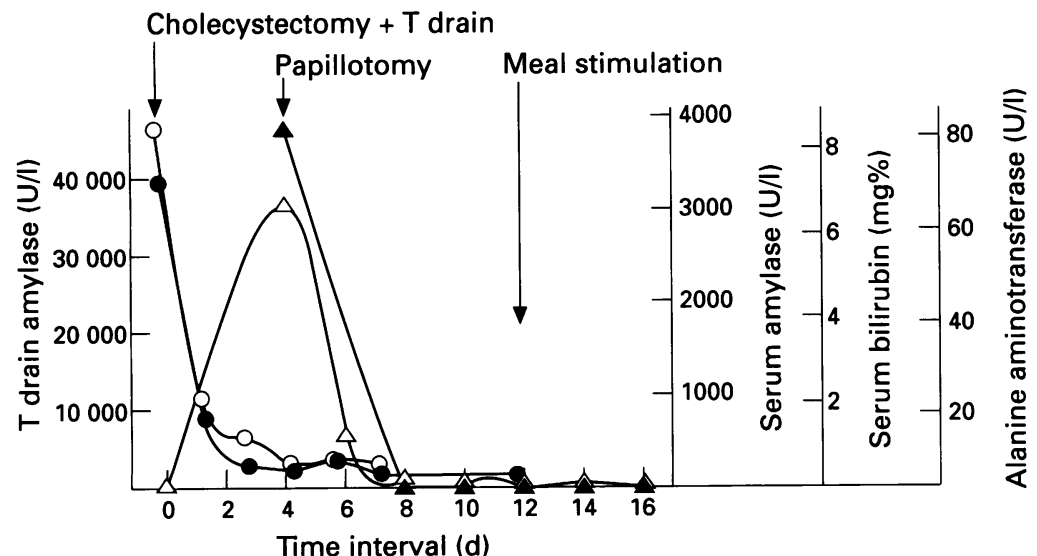

Figure 1: Time course and laboratory parameters from cholecystectomy to onset of pancreatitis. At the time of the initial admission (day 0) serum bilirubin (open circles, normal range $0 \cdot 1-1 \cdot 1 \mathrm{mg} \%$ ) and alanine aminotransferase (filled circles; normal range 5-24 U/l) were significantly raised while normal serum amylase (open triangles; normal range 0-120 U/T) showed absence of pancreatitis at this stage. Closed triangles show the activity of amylase in the $T$ drain bile from the time of referral to the gastroenterology unit. They decreased rapidly after removal of the stone and reflux of pancreatic juice into the bile ducts remained absent after meal stimulation.
It is now agreed that the passage of a stone from the gall bladder through the common bile duct can cause gall stone induced acute pancreatitis. ${ }^{1}$ The exact mechanism, however, by which the passing stone triggers pancreatitis is still a matter of debate. Opie suggested in 1901 that a migrating stone creates a channel between the pancreatic duct and the common bile duct when the stone is impacted at the narrowest part of its passage, the duodenal papilla. Through this common channel, Opie proposed, bile would regurgitate into the pancreas and thus trigger pancreatitis. ${ }^{2}$ This 'common channel theory' has been questioned because the secretory pressure in the pancreatic duct exceeds the pressure in the bile ducts. $^{3}$ Therefore, it might be expected that reflux towards the common bile duct rather than into the pancreas would result from a common channel situation. ${ }^{4}$ Moreover, perfusion of bile through the pancreatic duct at physiological pressure does not result in pancreatitis and the pancreatic duct remains unharmed. 56 Accordingly, it was proposed that obstruction of the pancreatic duct rather than biliopancreatic reflux may represent the critical event that triggers pancreatitis; this view is supported by recent experimental evidence. ${ }^{78}$ The findings that argue against the 'common channel theory' were mostly made in experimental animal models designed to mimic gall stone induced pancreatitis.

We report the case of a 22 year old woman with acute pancreatitis caused by a gall stone impacted at the duodenal papilla. In addition to this classic situation, identical to that reported by Opie in 1901, our patient had had cholecystectomy three days before the onset of pancreatitis and a $\mathrm{T}$ tube in the common bile duct was draining freely from the time of cholecystectomy to the onset of pancreatitis. Measurements of amylase activity in the $T$ drain fluid indicated significant reflux of pancreatic juice into the common bile duct up to the onset of pancreatitis and reversal of this regurgitation after endoscopic papillotomy and retrieval of the stone.

\section{Case report}

Right upper quadrant pain during childbirth was the first symptom of gall stone disease six months before admission to hospital of our 22 year old female patient. A second episode of biliary colic led to the referral to a community hospital. On admission the patient reported right upper quadrant pain, was found to have jaundice of the sclerae, and raised serum bilirubin concentration, and transaminase activity (Fig 1). Abdominal ultrasound showed 


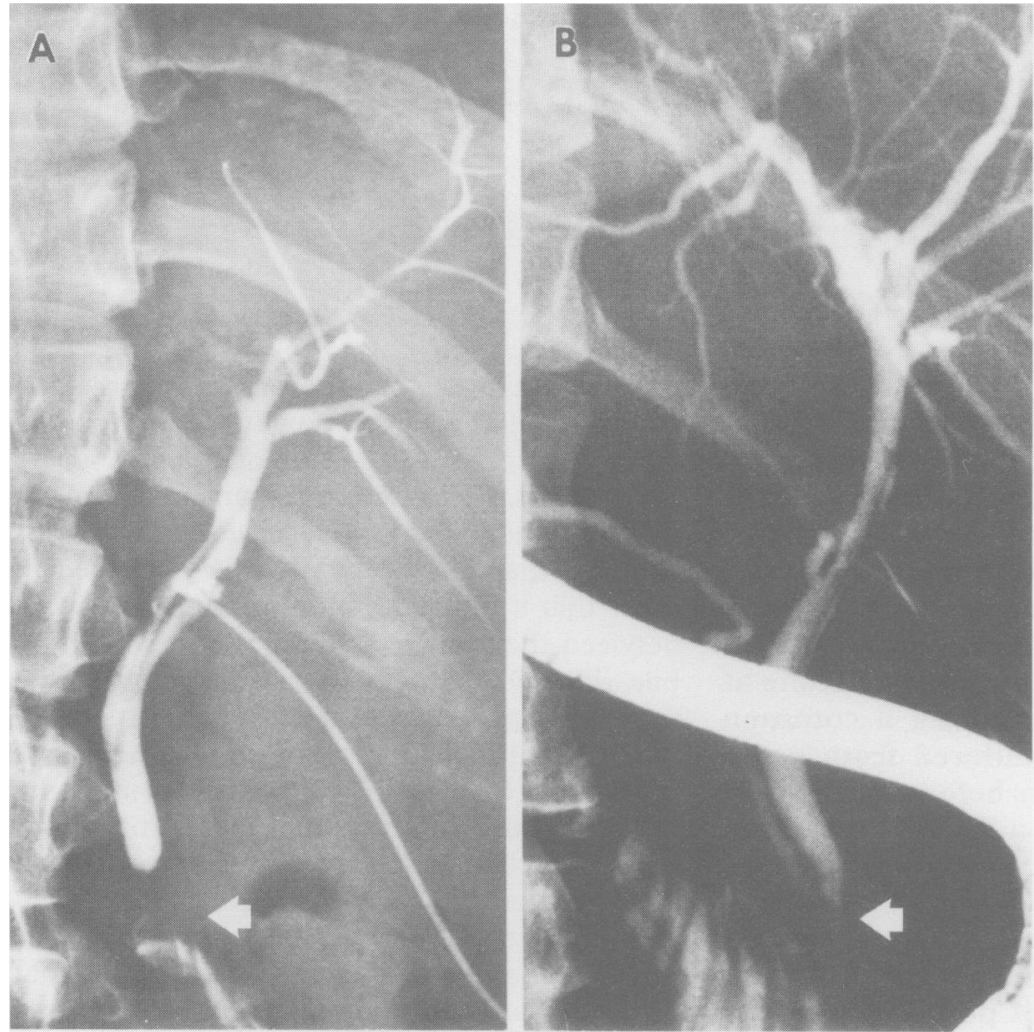

Figure 2: Retrograde contrast radiogram of the common bile duct. Note the prepapillary segment of the common bile duct (arrows) before papillotomy $(A)$ with a threadlike stricture, and after papillotomy (B) with restored patency of the duct.

multiple small stones in the gall bladder while the pancreas seemed completely normal. Serum amylase of $51 \mathrm{U} / 1$ (normal range 0-120 $\mathrm{U} / 1 ;$ Fig 1) and serum lipase of $54 \mathrm{U} / 1$ (normal range $0-190 \mathrm{U} / \mathrm{l}$ ) at this time were normal and excluded pancreatitis. Cholecystectomy was performed on the same day and the operating surgeon removed several small gall stones, 2-4 $\mathrm{mm}$ in size, from the common bile duct. Intraoperative cholangiogram, however, failed to show free passage of the contrast medium into the duodenum. Therefore a $\mathrm{T}$ drain was inserted into the common bile duct, to be left until recovery from the operation has occurred and endoscopic retrograde cholangiopancreatography at a later, unspecified date would have confirmed that passage from the biliary tract into the duodenum was patent. The patient's recovery was uneventful and bile was draining freely from the $T$ drain at a rate of 600 to $800 \mathrm{ml}$ per day.

Three days after surgery the patient developed epigastric pain radiating to the back. Serum amylase was increased to $3070 \mathrm{U} / 1$ and lipase to $>3000 \mathrm{U} / \mathrm{l}$. The patient was referred to our gastroenterology unit. An amylase of $46200 \mathrm{U} / \mathrm{l}$ in fluid from the $\mathrm{T}$ drain pointed to reflux of pancreatic juice into the common bile duct. On ultrasound and magnetic resonance imaging the pancreas was oedematous and the presence of a large bursal and paracolic effusion confirmed the diagnosis of acute pancreatitis. Endoscopically the papilla of Vater was inconspicuous but radiographic examination after injection of a contrast agent into the bile duct showed a short filling defect within the sphincter of Oddi (Fig 2). A small gall stone was recovered from the sphincter during endoscopic papillotomy. This rapidly alleviated the patient's symptoms and serum amylase and lipase concentrations decreased in parallel with pancreatic enzyme activity in the fluid from the biliary $T$ drain (Fig 1). The patient was given a light diet three days after papillotomy and tolerated it well. Ten days after papillotomy we studied whether physiological stimulation of the pancreas still induced reflux of pancreatic juice into the common bile duct. After an overnight fast pancreatic secretion was stimulated by giving the patient a standard test meal ( $400 \mathrm{kcal}$ of $38 \%$ carbohydrate, $41 \%$ fat, $21 \%$ protein) and fluid from the bile duct $\mathrm{T}$ drain was collected as previously described. ${ }^{8}$ The residual cholangiometric pressure in the common bile duct of $12 \mathrm{~cm} \mathrm{H}_{2} \mathrm{O}$ showed patency of the papilla. Amylase activity in fluid from the $\mathrm{T}$ drain at baseline and after meal stimulation was below detectable values (Fig 1). This showed that the regurgitation of pancreatic juice through a common channel into the bile duct, which had been seen in the presence of the impacted stone at the papilla, was now completely reversed after endoscopic papillotomy and retrieval of the stone.

\section{Discussion}

In 1901, Opie made two separate attempts to elucidate the mechanisms participating in the pathogenesis of acute gall stone induced pancreatitis. Both of his hypotheses were initially based on necropsy findings and both were later supported by experimental evidence obtained in Opie's laboratory. The initial hypothesis suggested that a gall stone that becomes impacted at the papilla occludes both the pancreatic duct and the bile duct. Consequently, obstruction of pancreatic outflow was proposed as the crucial event for the onset of acute pancreatitis. ${ }^{9}$ This impaired flow hypothesis' initially won little recognition and Opie's contribution to it has been nearly forgotten.

The second, alternative, hypothesis reported by Opie proposes that a channel between the pancreatic duct and the bile duct arises behind an impacted gall stone at the papilla and permits bile to regurgitate into the pancreas. The contact of the pancreatic epithelium with bile under pressure from the common duct was subsequently regarded as the triggering event for acute pancreatitis. ${ }^{2} 9$

This 'common channel theory' has found many followers despite a number of findings that are incompatible with its assumptions. One of the opposing arguments is based on the finding that pressure in the pancreatic duct exceeds biliary pressure, so that reflux into the biliary tract rather than into the pancreatic duct would occur in a common channel situation. ${ }^{34}$ Moreover, few patients with gall stone induced pancreatitis do in fact present with an impacted gall stone lodged at the papilla at the time of hospital admission. ${ }^{10} 11$ Even if a stone was situated within the sphincter, the terminal conduit into which the pancreatic duct and the common bile duct merge is often too short to 
allow for a common channel situation when an impacted stone is present. ${ }^{12}$ In about half the general population no common conduit is present at all, and in one fifth the pancreatic duct and the bile duct drain through a separate orifice into the duodenum. ${ }^{13}$

Despite these arguments, the 'common channel theory' has not lost its appeal. Its popularity was largely based on the comparative simplicity with which pancreatitis could be induced in a number of animal species by injecting bile (or various detergents and chemical agents) into the pancreatic duct. ${ }^{9} 14$ This apparent experimental confirmation, however, disregards two important circumstances. One is the pancreatic secretory pressure, which exceeds biliary pressure even after obstruction at the papilla as mentioned above. ${ }^{15}$ The second is the finding that bile, when perfused through the pancreas without the use of pressure in excess of physiological levels, does not cause pancreatitis and leaves the pancreatic duct unharmed. ${ }^{56}$ Experimental designs based on the chemical properties of bile in combination with unphysiological injection pressure are therefore poorly suited to reproduce the situation of a gall stone passing through the biliary tract.

The patient we present here developed acute pancreatitis resulting from the same events reported in Opie's necropsy case of 1901. In both cases, a gall stone was found impacted at the duodenal papilla and had apparently created a common channel between the pancreatic duct and the common bile duct. In our patient, however, the pre-existing presence of a $\mathrm{T}$ drain in the common bile duct permitted it to be shown that pancreatic enzymes had escaped through the common channel into the biliary tract and that bile flowed freely from the time of the cholecystectomy to the time of onset of pancreatitis and through the recovery period. Even if biliary secretory pressure in humans could theoretically rise to values in excess of pancreatic duct pressure, it could not have done so in this patient with an overflow drain in place. The time course of serum bilirubin and transaminase values in our patient further confirm that biliary pressure was not increased at the onset of pancreatitis. Instead we found excessive values of pancreatic enzyme activity in $T$ drain fluid collected before papillotomy that pointed to reflux of pancreatic juice into the common bile duct.

If, however, reflux of pancreatic juice into the biliary tract as seen in $\operatorname{dogs}^{34}$ and not regurgitation of bile into the pancreas results from an impacted stone, then Opie's 'impaired flow hypothesis'9 explains the pathogenesis of gall stone pancreatitis much more accurately than his "common channel theory.'

This particular case history shows that the argument whether bile reflux or pancreatic outflow obstruction causes pancreatitis is not purely an academic question but has important clinical implications. According to the 'common channel theory' the insertion of a common bile duct $\mathrm{T}$ drain at the time of cholecystectomy would have been entirely adequate to prevent bile reflux and the onset of pancreatitis in our patient. The 'impaired flow hypothesis', on the other hand, would have predicted that the restoration of pancreatic outflow was urgent and the only treatment needed. Had the impaired flow hypothesis' and not the 'common channel theory' determined the treatment for this patient the onset of acute pancreatitis could have been prevented. This assumption is in accordance with recent evidence from experimental ${ }^{16}$ and clinical studies, ${ }^{17} 18$ which show that an urgent relief of obstruction has also a beneficial therapeutic effect in biliary pancreatitis.

We conclude that the mechanisms participating in the development of pancreatitis in our patient contradict the hypothesis that reflux of bile into the pancreas is required for the onset of the disease. Our findings suggest that impairment of pancreatic outflow represents the triggering event for the onset of gall stone induced acute pancreatitis; confirming in humans what has been shown before in animal experiments.

The authors with to thank D K Nelson for valuable advice and discussion.

1 Acosta JL, Ledesma CL. Gallstone migration as a cause of acute pancreatitis. N Engl 7 Med 1974; 290: 484-7.

2 Opie EL. The etiology of acute hemorrhagic pancreatitis. fohns Hopkins Hosp Bull 1901; 12: 182-8.

3 Elliott DW, Williams RD, Zollinger RM. Alterations in the pancreatic resistance to bile in the pathogenesis of acute pancreatitis. Ann Surg 1957; 146: 669-82.

4 Mann FC, Giordano AS. The bile factor in pancreatitis. Arch Surg 1923; 6: 1-30.

5 Robinson TM, Dunphy JE. Continuous perfusion of bile protease through the pancreas. $7 A M A 1963 ; 183$ : $530-3$.

6 White TT, Magee DF. Perfusion of the dog pancreas with bile without producing pancreatitis. Ann Surg 1960; 131: 245-50.

7 Lerch MM, Saluja A, Rünzi M, Dawra R, Saluja M, Steer ML. Pancreatic duct obstruction triggers acute necrotizing pancreatitis in the opossum. Gastroenterology 1993; 104: 853-61.

8 Hernandez CA, Lerch MM. Sphincter stenosis and gallstone migration through the biliary tract. Lancet 1993; 341: 1371-3.

9 Opie EL. The relation of cholelithiasis to disease of the pancreas and to fat-necrosis. Fohns Hopkins Hosp Bull 1901; 12: 19-21.

10 Stone HH, Fabian TC, Dunlop WE. Gallstone pancreatitis. Biliary tract pathology in relation to time of operation. Ann Surg 1981; 194: 305-12.

11 Kelly TR, Wagner DS. Gallstone pancreatitis: a prospective randomized trial on the timing of surgery. Surgery 1988; 104: $600-5$.

12 Sterling JA. The common channel for bile and pancreatic ducts. Surg Gynecol Obstet 1954; 98: 420-4.

13 DiMagno EP, Shorter RG, Taylor WF, Go VLW. Relationship between pancreaticobiliary ductal anatomy and pancreatic ductal histology. Cancer 1982; 49: 361-8

14 Aho HJ, Nevalainen TJ. Experimental pancreatitis in the rat. Ultrastructure of sodium taurocholate-induced pancreatic lesions. Scand $\mathcal{f}$ Gastroenterol 1980; 15: 417-24.

15 Menguy RB, Hallenbeck GA, Bollman JL, Grindlay JH. Intraductal pressures and sphincteric resistance in canine pancreatic and biliary ducts after various stimuli. Surg Gynecol Obstet 1958; 106: 306-20.

16 Rünzi M, Saluja AK, Lerch MM, Dawra R, Nishino $H$, Steer ML. Early ductal decompression prevents the progression of biliary pancreatitis: an experimental progression of biliary pancreatitis: an experimental study in

17 Neoptolemos JP, London NJ, James D, Carr-Locke DL Bailey IA, Fossard DP. Controlled trial of urgent endoscopic retrograde cholangiopancreatography and endoscopic sphincterotomy versus conservative treatmen for acute pancreatitis due to gallstones. Lancet 1988; ii 979-83.

18 Fan ST, Lai ECS, Mak FPT, Lo CM, Zhang SS, Wong J Early treatment of acute biliary pancreatitis by endoscopic papillotomy. N Engl f Med 1993; 328: 228-32. 